\title{
A COUNTEREXAMPLE TO A THEOREM OF CHASE
}

\section{PHILLIP GRIFFITH ${ }^{1}$}

1. Introduction. Throughout this note all groups are abelian. The notation is that of [2]. If $G$ is a torsion free group and $H$ is a subgroup of $G$, we shall use the symbol $H_{*}$ to denote the minimal pure subgroup of $G$ containing $H$. Also, the symbols $\sum$ and + will be used for direct sums; whereas the subgroup of a group $G$ generated by its subsets $S$ and $T$ will be denoted by $\{S, T\}$.

In answer to a question of Nunke, Chase [1] proved incorrectly the following theorem and its immediate corollary.

Theorem (ChASE). Let $G$ be a torsion free group, and let $F_{0}$ and $F_{1}$ be pure free subgroups of $G$ of rank $\alpha$ and $\beta$ respectively where $\alpha<\beta$ and $\beta$ is infinite. Then there exists a pure free subgroup $F$ of $G$ such that $F_{0}$ is a direct summand of $F$ and rank $(F)=\beta$.

Corollary (CHASE). If $X_{0}$ and $X_{1}$ are maximal pure independent subsets of a torsion free group $G$, one of which is infinite, then both have equal cardinality.

In fact, these results are false as stated. We shall demonstrate this by exhibiting a counterexample to the corollary. Chase's proof is correct when $\alpha$ and $\beta$ are both infinite, but there is a flaw when $\alpha$ is finite and $\beta$ is infinite. We construct a countable torsion free group $G$ with maximal pure independent subsets $X_{0}$ and $X_{1}$ such that $\left|X_{0}\right|=1$ and $\left|X_{1}\right|=\boldsymbol{\aleph}_{0}$. Letting $F_{0}$ and $F_{1}$ be the pure free subgroups generated by $X_{0}$ and $X_{1}$ respectively, we immediately observe that Chase's theorem and corollary do not hold for $G$. Finally, we remark that a careful study of Chase's proof yields that counterexamples to the theorem can occur only when $\alpha$ is finite and $\beta$ is at most countable.

2. Counterexample. We form the direct sum $A=Q+D$ where $Q$ denotes the rational numbers and $D$ is the minimal divisible group containing a free group $F$ of rank $\boldsymbol{\aleph}_{0}$. Let $p_{1}<p_{2}<\cdots$ be the usual ordering of the primes. Define $\left[b_{1}, b_{2}, \ldots\right]$ to be the collection of all nonzero elements $x$ of $F$ such that $x$ generates a pure cyclic subgroup of $F$. Note that no $b_{n}$ is divisible in $F$ by any prime. Select a nonzero element $y$ in $Q$ and set $B=\left[p_{n}^{-k}\left(y+b_{n}\right) \mid n=1,2, \cdots\right.$ and $k=1,2, \cdots]$. Let $G$ be the subgroup of $A$ generated by the group

Received by the editors March 13, 1967.

1 The author wishes to acknowledge support by the National Aeronautics and Space Administration Grant $\mathrm{NsG}(\mathrm{T})-52$. 
$\{y\}+F$ and the set $B$. We show that $\{y\}$ and $F$ are pure subgroups of $G$.

Observe that a typical element $g \in G$ has the form

$$
g=f+t y+\sum_{i=1}^{r} m_{i}\left(y+b_{i}\right),
$$

where $f \in F, t$ is an integer, $m_{i}=t_{i} p_{i}^{-k_{i}}$, and $t_{i}$ is an integer such that $\left(t_{i}, p_{i}\right)=1$ for $i=1, \cdots, r$. If $n g \in\{y\}$ for some nonzero integer $n$, then $n f+n \sum_{i=1}^{r} m_{i} b_{i}=0$ since $A=Q+D$. Therefore, $f=-\sum_{i=1}^{r} m_{i} b_{i}$. Set $u_{i}=\prod_{e=1 ; e \neq i}^{r} p_{e}^{k_{e}}$. It follows that $\left(p_{i}, u_{i}\right)=1$ and that $u_{i} m_{e}$ is an integer for $i \neq e$. Therefore, $-u_{i} m_{i} b_{i}=u_{i} f+\sum_{e=1 ; \notin \neq i}^{r} u_{i} m_{e} b_{e} \in F$. Our definition of $b_{i}$ implies that $u_{i} m_{i}$ must be an integer for $i=1, \cdots, r$. Since $\left(p_{i}, u_{i}\right)=1$ and since $m_{i}=t_{i} p_{i}^{-k_{i}}$, it follows that $u_{i} m_{i}$ is an integer if and only if $m_{i}$ is an integer for $i=1, \cdots, r$. Hence, $g=\left(t+\sum_{i=1}^{r} m_{i}\right) y \in\{y\}$. Thus, $\{y\}$ is a pure subgroup of $G$. If $n g \in F$, then $n t y=\left(-n \sum_{i=1}^{r} m_{i}\right) y$ since $A=Q+D$. Therefore, $t y$ $=\left(-\sum_{i=1}^{r} m_{i}\right) y$ implies that $-\sum_{i=1}^{r} m_{i}$ is an integer. Hence, as observed above, this implies that each $m_{i}$ is an integer. Therefore, $g=f+\sum_{i=1}^{r} m_{i} b_{i} \in F$ and $F$ is a pure subgroup of $G$.

Expressing $F$ as $F=\sum_{i<\omega}\left\{x_{i}\right\}$, we have that $\left[x_{i}, x_{2}, \cdots\right]$ is a pure independent subset of $G$, since $F$ is a pure subgroup of $G$. Let $X_{1}$ be a maximal pure independent subset of $G$ containing $\left[x_{1}, x_{2}, \ldots\right]$. Then $\left|X_{1}\right|=\boldsymbol{\aleph}_{0}$. Since $\{y\}$ is a pure subgroup of $G$, $X_{0}=[y]$ is a pure independent subset of $G$. We establish that $X_{0}$ is a maximal pure independent subset of $G$ by showing that $\{y, g\} *$ is not free for any $g \in G$ which is independent from $y$.

Let $g \in G$ be such that $g$ and $y$ are independent. Since $A /(\{y\}+F)$ is torsion, there is a nonzero integer $n$ and an integer $m$ such that $n g=m y+f$ where $f \in F$. The independence of $y$ and $g$ implies that $f \neq 0$. Therefore $n g-m y=f \in\{y, g\}_{*}$. For some positive integers $u$ and $v$, we have that $u b_{v}=f$. Therefore $b_{v}$ must be an element of $\{y, g\}_{*}$. Since $y+b_{v} \in\{y, g\}_{*}$ and since $\{y, g\}_{*}$ is a pure subgroup of $G$, then $p_{0}^{-k}\left(y+b_{v}\right) \in\{y, g\}_{*}$ for $k=1,2, \cdots$. Thus $\{y, g\}_{*}$ cannot be free.

\section{REFERENCES}

1. S. Chase, "On group extensions and a problem of J. H. C. Whitehead" pp. 173193, in Topics in abelian groups, Scott, Foresman and Co., Chicago, Ill., 1963.

2. L. Fuchs, Abelian groups, Akad. Kiad6, Budapest, 1958.

UNiversity OF Houston 